\title{
Near Infrared Spectroscopy and Spiroergometry Testing in Crossfit
}

\author{
Petr Schlegel, Jan Hiblbauer, Adrián Agricola \\ University of Hradec Králové, Faculty of Education, Hradec Králové, Czech Republic
}

\begin{abstract}
PURPOSE: CrossFit is a young sport discipline which offers varied strength and endurance training through complex exercises. Currently there are relatively few studies focusing on performance analysis in terms of the physiological response of organism. The aim of the research was to verify near infrared spectroscopy (NIRS) in combination with spiroergometry as a functional means for specific load testing in CrossFit and to find out what physiological changes occur in CrossFit.

METHODS: Elite crossfitter (male, age 20, body height $185 \mathrm{~cm}$, body weight $87 \mathrm{~kg}$ ) formed part of this study. Two Moxy sensors (placed on the vastus lateralis muscle and the intercostal muscles) and chest (strap) heart rate (HR) sensor were used for obtaining the data. The Cortex MetaMax 3BR2 system was used for portable spiroergometry. The AMRAP method (as many repetitions as possible) was used for testing. The selected test consisted of (1) 10 Deadlifts, 100 kg, 15 Assault Air Bike Calories; (2) 12 lunges (with two 20 kg Kettlebell), 10 push-ups, 8 ring pull up; (3) 20 SkiErg Calories, 1050 kg back squat, 10 toes to bar.

RESULTS: The testing has confirmed that breathing functions and muscle oxidation can be well observed under load in given exercises and movements. It has been confirmed that CrossFit provides a very varied load to which the organism must respond immediately. The strength load changes, causing deflection in $\mathrm{SmO} 2$ and VCO2 which consequently imposes demands on the respiratory component.

CONCLUSIONS: This is the first study which monitors the load in the combination of strength and endurance load through crossfit elements. Based on our result, it seems that linking NIRS and spirometry is a suitable combination for a comprehensive analysis of the athlete not only for CrossFit. The information obtained can be applied in practice in the context of optimal training load settings.
\end{abstract}

Keywords: CrossFit, near infrared spectroscopy, spiroergometry, performance

\section{INTRODUCTION}

CrossFit has become a world-wide phenomenon over the last 10 years. Originally intended as training system, it has gradually developed into a full-fledged sport discipline. The goal of CrossFit is to comprehensively develop physical fitness (Glassman, 2007), thus influencing also health. It is a very variable high intensity exercise involving functional movements. The source of exercises and training approaches are three modalities (weightlifting, gymnastics, metabolic conditioning), which are represented to the same extent. The result is so called WOD (workout of the day), which consists of one, two or all three modalities and usually lasts 5-20 minutes. The nature of the exercise can be described as High-Intensity Functional Training (Feito, 2018), which includes multi-joint exercises with the involvement of major muscle groups. At the same time, the exercise takes place at a high heart rate and is usually relatively short. It is a functional tool for the development of metabolic and cardiorespiratory parameters. The purpose of CrossFit, however, is also a targeted effect on strength abilities including work with one's own bodyweight as well as 
external load. Although there is a concern about the safety of CrossFit (rhabdomyolysis, risk of injury, etc.), it has been confirmed that it meets the requirements stated in the American College of Sports Medicine. Drum (2017) notes that there is no serious risk with properly adjusted load and suitably selected regeneration. There is also a higher oxidative stress during exercise, but it is comparable with running load (Kliszczewicz, 2015). Even in terms of muscle damage indicated through creatine kinase marker, CrossFit does not stand out among other sport disciplines in terms of training load (Heavens, 2014).

High load in CrossFit was confirmed by Fernández, Solana, Moya, Marin, and Ramón (2015), where physical indicators were monitored. After 'Cindy' and 'Fran' WODs, the athletes achieved high levels of heart rate, VO2max and lactate. Also Perciavalle (2016) reaches similar conclusions except that the monitored workout consisted of a rowing machine and a big dumbbell. The mean lactate values measured after exercise were $13.8 \mathrm{mmol} / \mathrm{L}$, which mean a high intensity. The Tibana research (2016) was conducted under similar load and achieved similar conclusions. Moreover, it came to the conclusion that, after two demanding training days, the athletes have shown high cytokine values; therefore, the research recommended optimizing intensity in training load.

The physiological response after a CrossFit exercise was examined also by Maté-Muñoz (2018). Here the load consisted of separate gymnastics ('Cindy'), metabolic conditioning (double skip rope jumps), weightlifting (power cleans). Individual modalities differed from each other, for instance they reached lower values of maximum heart rate in power clean. However, in all cases they exceeded the lactate level of $10 \mathrm{mmol} / \mathrm{L}$ and evaluated the activities with a high RPE. CrossFit performance is determined by many aspects and so far it is not quite clear which of them are key for achieving success, resp. high performances. In terms of physiological determinants, it seems that VO2max and anaerobic peak power are very important (Bellar, 2015; Dexheimer, 2019). Nevertheless, also the level of strength abilities occupies an important position and the exact determination of the effect of specific parameters is not clear (Butcher, 2015). Similarly, as in other sport disciplines, it is important to find a suitable test means to determine performance and functional limitation.

Near infrared spectroscopy (NIRS) is an imaging technique developed in the 1970ies that has expanded during the 1990. Originally focused on brain research, it was later gradually introduced also in other fields of science (Ferrari \& Quaresima, 2012). It is a non-invasive imaging of oxidation and hemodynamics in the muscle using infrared radiation. It was used for the first time to monitor haemoglobin resaturation in quadriceps muscles in racing rowers (Chance, 1992). Since then, it has been applied to groups of runners, cyclists, rugby players, swimmers, etc. as well as to various muscle groups (Perry \& Ferrarri, 2017). So far, only one study has been aimed at examining the effect of the strength load on lower limbs; the means was isolated knee extension motion (Paradis-Dechênes, 2016). The response to local isometric loading is presented by Freyer (2015) on climbers. Currently, there is no known research which would monitor muscle oxidation through the means of complex training load or combination of strength and endurance loads.

Moxy oxygen monitor is a device which measures, among others, local oxygen saturation ( $\mathrm{Sm02}$ ) and total haemoglobin mass ( $\mathrm{tHb}$ mass), value of which corresponds to the current muscle blood flow. The validity and reliability of the device has been confirmed on a sample of high performance cyclists (Crum, 2017). It has also proven itself in comparison with a similar PortaMon device. However, the authors add that the comparison of results from various devices may differ (McManus, 2018).

Spiroergometry is a tool for a qualitative and quantitative assessment of cardiovascular, pulmonary and metabolic responses to exercise. Measuring oxygen consumption, carbon dioxide production, minute ventilation and heart rate provides significant diagnostic and prognostic information across a wide range of scientific fields (Wonisch, 2003). The most important variable 
in spiroergometry is the maximum oxygen consumption (VO2max). It defines the capacity of cardiopulmonary system and provides an objective estimate of physical fitness. Minute ventilation (VE) consists of breathing frequency and tidal volume (Vt). The VE maximum value (VEmax) during exercise is important to achieve a high VO2max value. In addition, the ventilation threshold and 'respiratory compensation point' can be determined as submaximal fitness parameters. The methodology of spiroergometric examination is based on the analysis of the inhaled and exhaled air composition and serves to determine the functional response of the organism to the load (Corrà, 2018). Oxygen consumption and exhaled carbon dioxide levels are monitored during exercise; pulmonary ventilation and heart rate are also monitored. Repeated testing is an important diagnostic parameter in monitoring the adaptation of the organism to training load. Spiroergometry is, in contrast with our research, commonly performed in standardized conditions with precisely defined test parameters.

The physiological reaction of the organism to the load is an important element for optimal adjustment of training intensity, therefore the selection of specific methods, etc. CrossFit uses a very variable load, for which acute physiological responses are not yet clear. The aim of the study was to monitor physiological changes through CrossFit training and to verify whether NIRS in combination with spiroergometry is a suitable means for such testing.

\section{METHODS}

\section{Participant}

The presented case study is based on the results of the diagnostic process of an elite crossfiter 4 year experience, result in CrossFit OPEN 2019 in the Czech Republic among top 20, (male; age 20 years; body height $185 \mathrm{~cm}$; body weight $87 \mathrm{~kg}$ ). The tested athlete had no special preparation before testing. The athlete was instructed to follow his normal diet and standard daily regimen. He had a rest the previous day before testing.

\section{Material}

To obtain the research data, two Moxy sensors were used which were placed on the body of the athlete (on the vastus lateralis muscle and the intercostal muscles) and chest (strap) heart rate sensor (HR). The Cortex MetaMax 3BR2 system was used for portable spiroergometry.

\section{Procedure}

The selected test consisted of three six-minute sections, between which there were two-minute breaks.

(1) 10 Deadlifts, $100 \mathrm{~kg}, 15$ Assault Air Bike Calories ${ }^{1}$

(2) 12 lunges (with two $20 \mathrm{~kg}$ Kettlebell), 10 push-ups, 8 ring pull up

(3) 20 SkiErg Calories, $1050 \mathrm{~kg}$ back squat, 10 toes to bar

The AMRAP method (as many repetitions as possible) was used, where the athlete tried to do the maximum repetition of given exercises within a set time limit. The breaks or pauses were organized by the athlete himself, as well as exercise speed or pace. The break between the intervals was not organized; the athlete did not have to rest actively. He got time for individual warm-up and preparation before the test.

\footnotetext{
Calories are the unit of measurement used for cardio machines as Assault Air Bike, SkiErg, rowing machine.
} 
The methodology of the research is based on the evaluation of individual organ energy systems through specific parameters:

(1) Breathing $-\mathrm{EV}$ (minute ventilation $-\mathrm{L} / \mathrm{min})=\mathrm{BF}$ (breathing frequency $-1 / \mathrm{min}) \times \mathrm{VT}$ (tidal volume - L); \%BR (breathing reserve percentage) - the amount of remaining breathing capacity (2) Cardiovascular system - HR (heart rate), VO2 (oxygen consumption/HR (pulse oxygen), tHb (total haemoglobin mass)

(3) Metabolism - indicative value of the level of aerobic/anaerobic metabolism using Respiratory Equivalent Ratio $(\mathrm{RER}=\mathrm{VCO} 2 / \mathrm{VO} 2)$;

(4) carbon dioxide production (VCO2); METS - metabolic equivalents - multiple of resting energy expenditure $(\mathrm{VO} 2=3.5 \mathrm{ml} / \mathrm{kg} / \mathrm{min})$

Muscle tissue - $\mathrm{SmO} 2$ (muscle oxygen saturation), $\mathrm{tHb}$

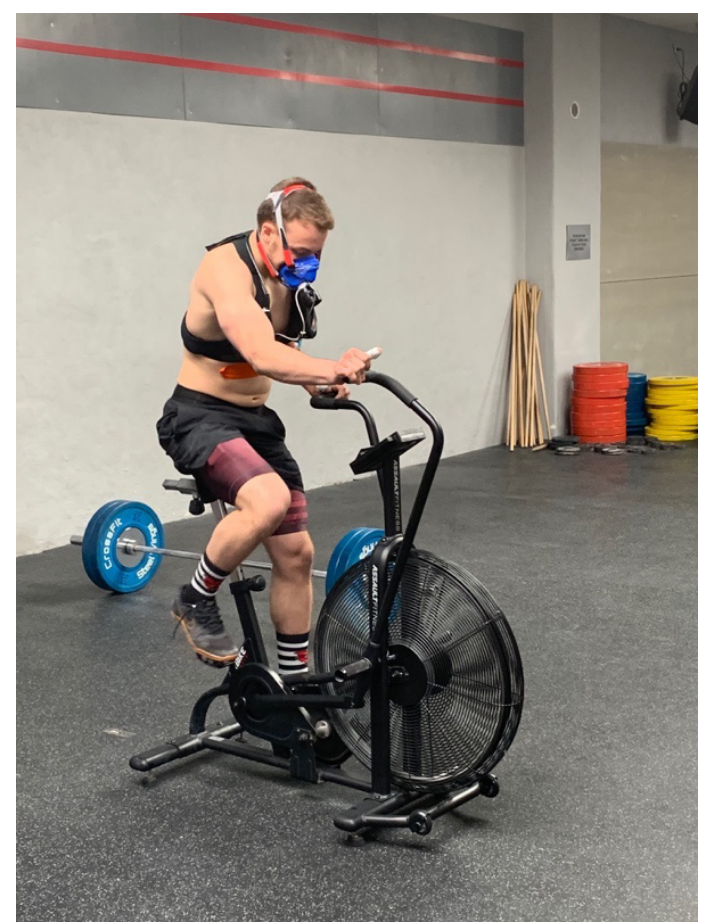

Fig. 1: Assault Air Bike

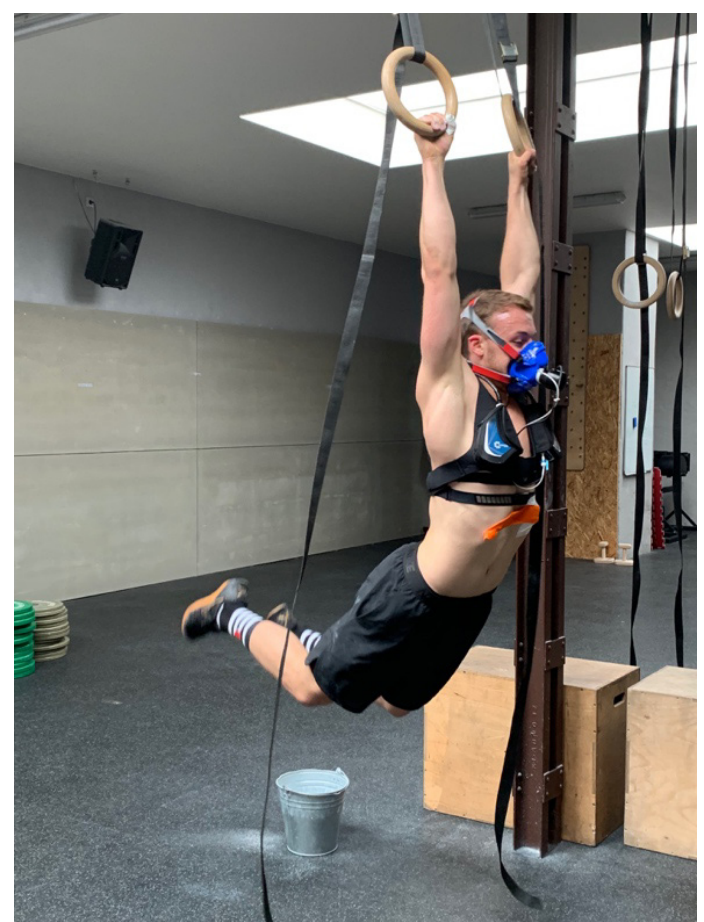

Fig. 2: A ring pull up

\section{RESULTS}

The results showed high heart rate values above the level of anaerobic threshold, while also the rate of their decrease was monitored. Heart pulse rate corresponded to breathing functions. These values were significantly reduced during rest. Figure 3 shows the regeneration process by individual intervals and the speed of return of muscle oxygenation. 


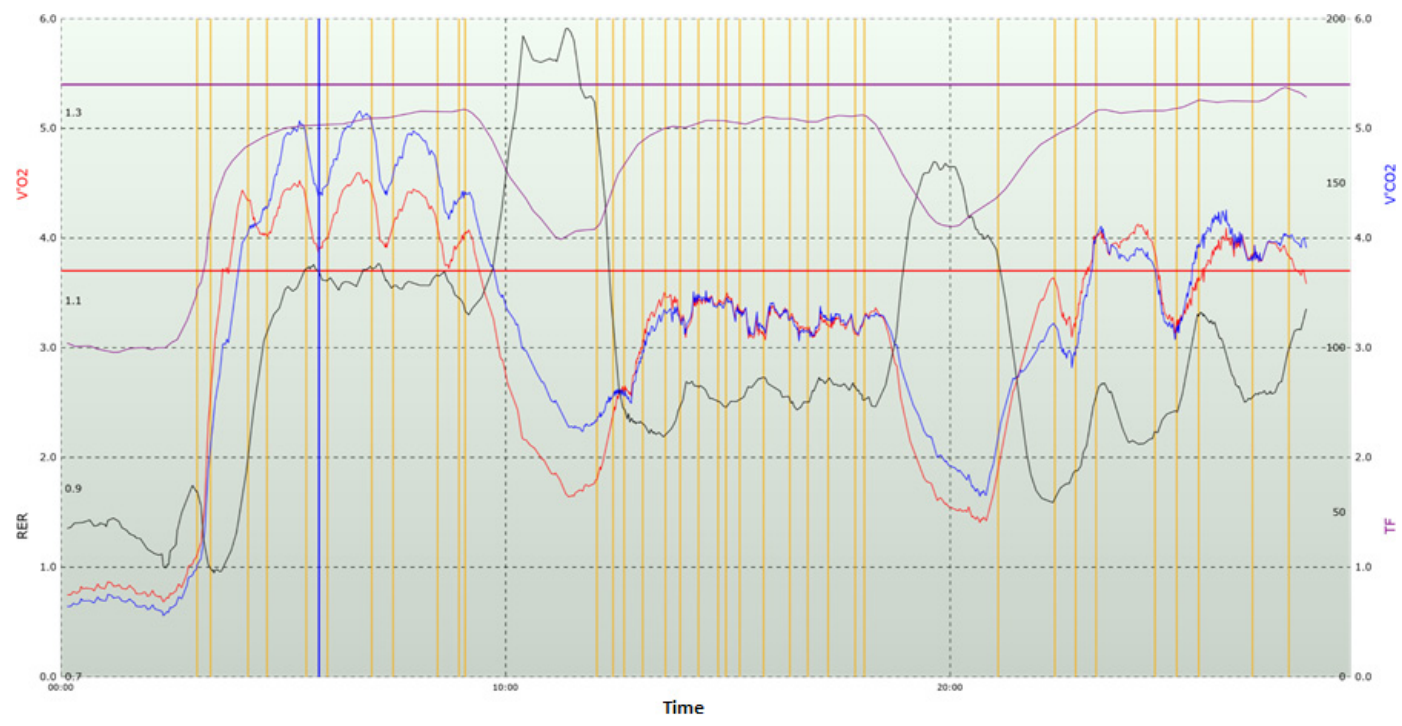

Fig. 3: VO2, RER, VCO2 and HR values

Note: V02 - oxygen consumption; RER - Respiratory Equivalent Ratio; VCO2 - carbon dioxide production; HR - heart rate

The first interval composed of a power exercise aimed at a large muscle group followed by an intense endurance performance led to high absolute levels of $\mathrm{O} 2$ compared to the second interval which was composed of exercises with a low external load. During the load, especially $m$. vastus lateralis showed a lower blood supply associated with reduced oxygen delivery to the muscle. In the following phase, however, there was a good recovery (whether on the Assault Air Bike or SkiErg - in both cases, the lower limbs needed to do some work; nevertheless, it was sufficient to improve the oxidative supply).

The changes of VCO2 can be monitored at each interval. The load on the lower limbs was lower in the second part, but there were still significant changes. Compared to the first interval, this part was less metabolically demanding. The connection of isotonic and isometric work of the quadriceps in lunges and push-ups kept the oxidation at lower values. Even though the push-up concentrates on the upper half of the body, it was not an ideal means for the acute full recovery of the lower limb.

The third interval already clearly shows fatigue accumulation from the previous parts. The athlete maintained a high intensity associated again with lower oxygen delivery in both muscles. However, it did not reach such values as in the first part, which dramatically loaded the lower limbs. Good tolerance to anaerobic load and to strength and conditioning load can be observed during testing. 


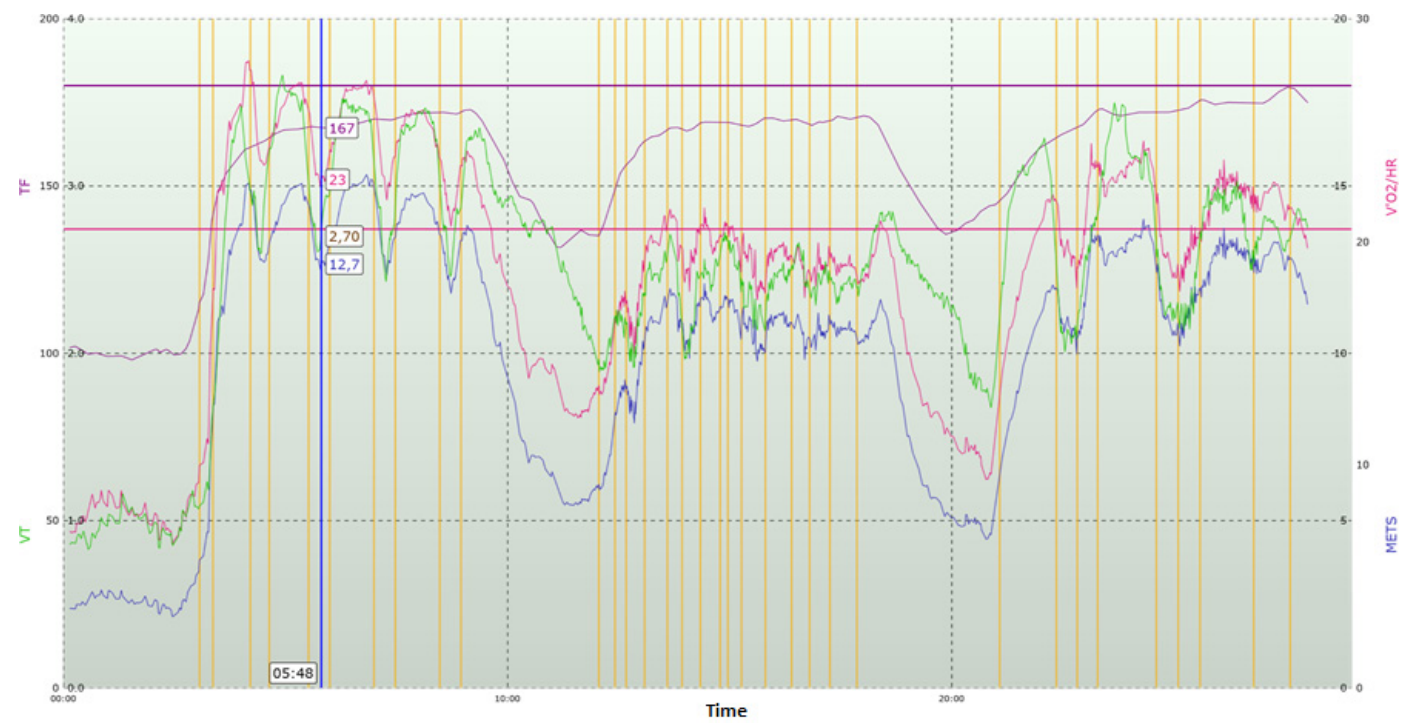

Fig. 4: VT, VO2/HR, METS and HR values

Note: VT - tidal volume; V02/HR - pulse oxygen; METS - metabolic equivalents; HR - heart rate

Figure 4 shows, among others, that HR may not always correspond to the metabolic demand of the exercises. All three intervals take place in comparable HF (160-170/min), but the values of RER, VO2, VCO2 and METS differ significantly, thus determining different metabolic demands. Each interval was different not only in intensity, but also in structure, which can be clearly seen in different graph curves.

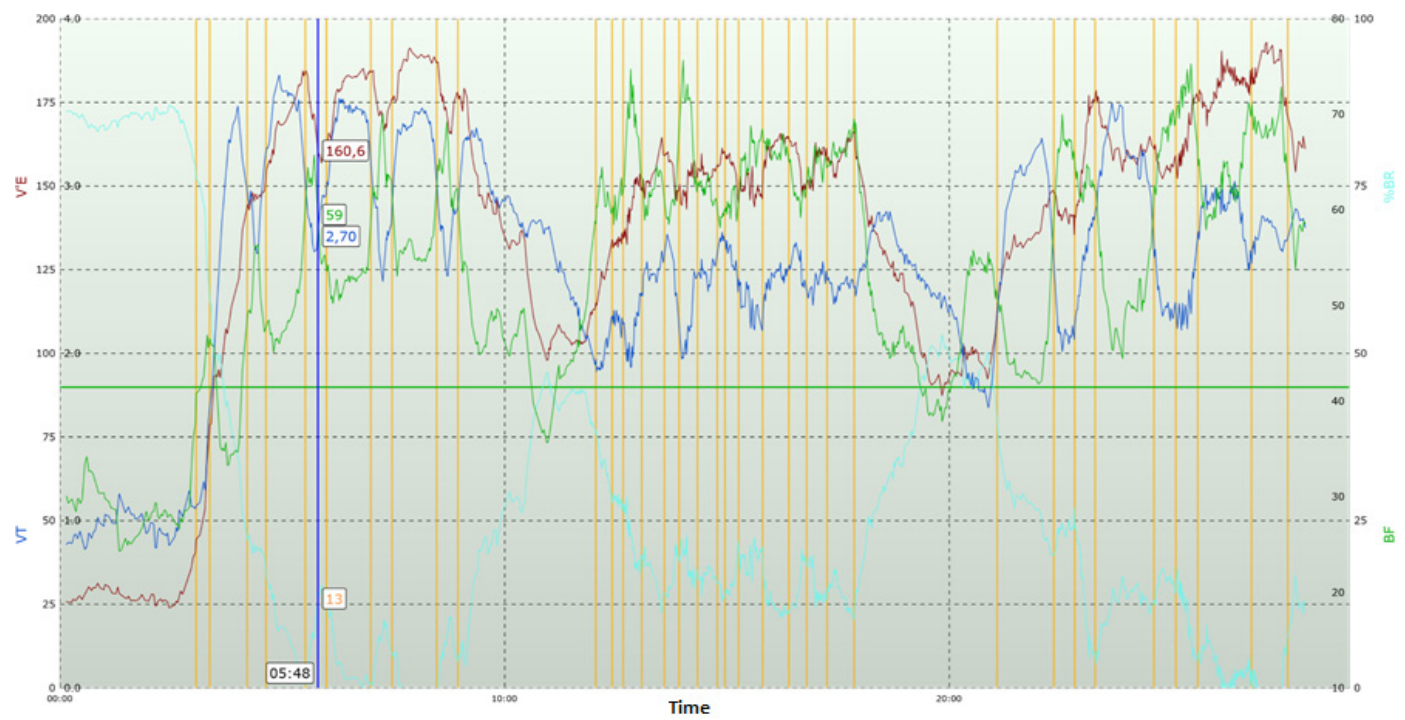

Fig. 5: $V T, V E, B F$ and \%BR values

Note: VT - tidal volume; VE - minute ventilation; $\mathrm{BF}$ - breathing frequency, $\% \mathrm{BR}$ - breathing reserve percentage 
It can be clearly seen from Figure 5 that, during the first interval after 2.5 minutes, the athlete had already reached the phase in which he used his ventilation capacity almost up to $100 \%$. It is also possible to observe changes in breathing frequency and in inhalation / exhalation quality during the intervals. The specific load for instance in deadlift or exercises on the horizontal bar and rings had led to a change in breathing pattern and partly also to an occasional decrease in respiratory depth.

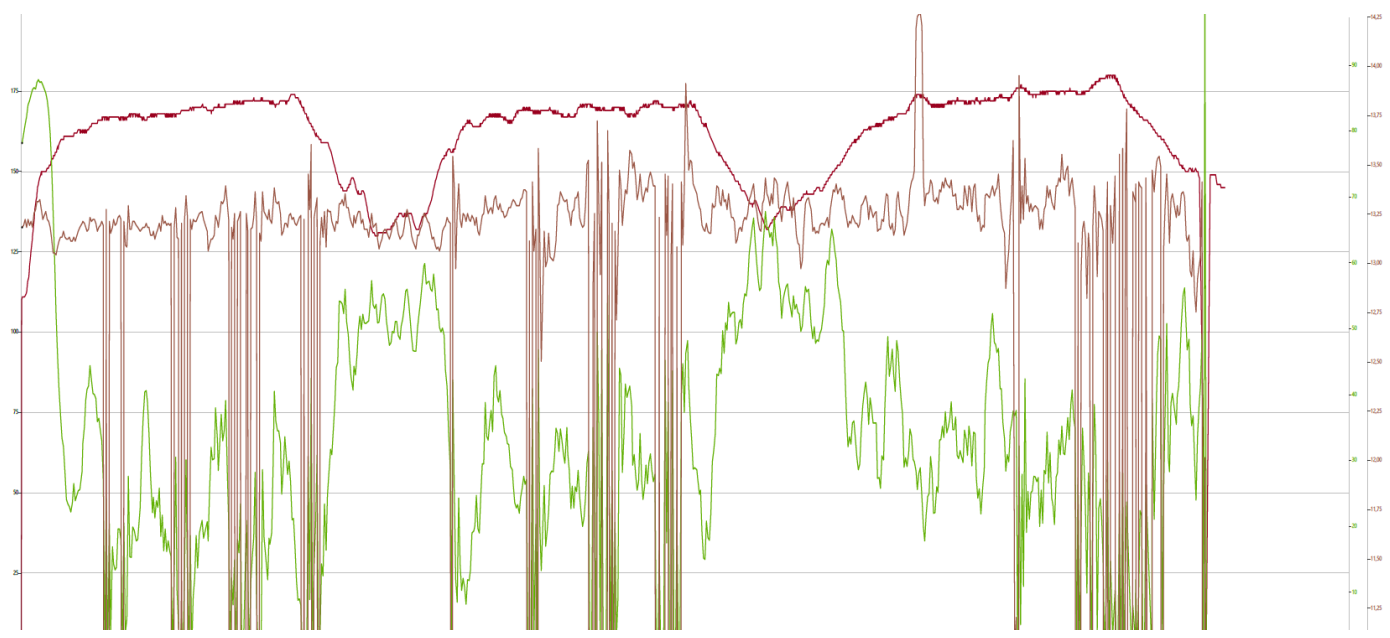

Fig. 6: Moxy record from the intercostal muscle

The record shows the effect of the load on respiratory muscles, which is, among others, related to the detection of the risk of metaboreflex. The green curve shows the course of oxygen utilization in the muscle, the brown one the rate of blood flow, the red curve corresponds to HR.

\section{DISCUSSION}

This is the first study which monitors the load in the combination of strength and endurance load through crossfit elements. Isolated muscle work can be seen in Paradis-Dechênes (2016) or Freyer (2015) with a corresponding change of haemodynamics. Nevertheless, it is more important for practical use to apply a load similar to a specific sport performance. The testing has also confirmed that breathing functions and muscle oxidation can be well observed under load in given exercises and movements. Most studies deal with the physiological response in cyclic movements like running, rowing, etc. (Perrey \&Ferrari, 2017), from which it is clear that they represent a legitimate tool for monitoring the use of oxygen in the muscle. Here the concept of combined load was applied and, at the same time, specific tools were used. Similarly, however, we note large changes in oxygen saturation during intense load.

It has been confirmed that CrossFit provides a very varied load to which the organism must respond immediately. The strength load changes, causing deflection in $\mathrm{SmO} 2$ and $\mathrm{VCO} 2$ which consequently imposes demands on the respiratory component. The position of the body is not constant either, which has resulted in a change in spirometric parameters for instance in pull ups or toes to bar. During rest periods, an increase in RER was noted (RER $>1.0$ indicates significant anaerobic metabolism) which can be explained by the intensity of activity in the anaerobic zone and by good individual fitness predisposition of the athlete. The athlete used almost his maxi- 
mum ventilation capacity, which indicates an automatic response to the load at a given intensity, where there is a large consumption of $\mathrm{O} 2$ and thus also the production of $\mathrm{CO} 2$, of which the body has to automatically dispose (extended spinal cord reflex). Higher breathing capacity is a positive prerequisite for a high intensity of endurance performance. High demands on the work of respiratory muscles can be linked with their fatigue and consequent negative effect on $\mathrm{CO} 2$ exhaling, which is main factor determining centrally perceived fatigue (Guynet \& Bayliss, 2015). Measurements have shown a significant influence of breathing parameters on performance in CrossFit; therefore, it seems important to monitor the performance of respiratory muscles, which are susceptible to fatigue, but can also be trained like other striated muscles (HajGhanbari, 2013).

HR is a commonly used intensity indicator which, however, has its limitations. There is a delayed response to the load (in tens of seconds) in contrast with NIRS (Morgan \& Mora, 2017). The measurements show that there is no direct link between HR and the observed metabolic variables (unlike Maté-Muñoz, 2018). Therefore, HR cannot be perceived as an accurate indicator of body's overall workload during strength-endurance exercises (Born, 2017).

The purpose of testing was to simulate the environment which is identical with the load in CrossFit. The selection of exercises had to be adapted to the conditions. For instance, the measuring devices (see Fig. 1) did not allow the inclusion of Olympic weightlifting or demanding gymnastic exercises which are commonly used in CrossFit. The intervals were put together according to the combination of various modalities to load the athlete in very different ways and to change the strength and endurance demand.

Unlike laboratory tests, where the objective is to expose the body to increasing work intensity, the performance here has moved, from the very beginning, to the area close to the anaerobic threshold. It is therefore not possible to use routine procedures for the evaluation.

It can be stated from the results that the athlete has a tolerance to high load exceeding the anaerobic threshold, in which (in contrast with purely endurance activities) he has to be able to maintain an optimum concentration level for performing movements demanding on coordination.

\section{CONCLUSION}

It is possible to analyse very well from the results of the test the overall performance of the athlete and read the physiological response to strength, endurance or combined stimuli in CrossFit. This test gives a good feedback on the response of organism to the load, which may well be used for the subsequent optimization of training. Joining NIRS and spirometry seems to be a good combination for the comprehensive body load analysis: the use of the method could be beneficial also for other sport disciplines. At the same time, it is confirmed that the parameters from both measurements are related and, together with heart rate values, they create the overall picture of the organism's response during testing. Due to the originality of the research, more studies are needed to confirm some conclusions (e.g. magnitude of drop and return smo2 rate, RER values or importance of respiratory rate for CrossFit performance).

\section{REFERENCES}

Bellar, D., Hatchett, A., Judge, L., Breaux, M., \& Marcus, L. (2015). The relationship of aerobic capacity, anaerobic peak power and experience to performance in CrossFit exercise. Biology of Sport, 32(4), 315-320.

Born, D., Stöggl, T., Swarén, M., \& Björklund, G. (2017). Running in Hilly Terrain: NIRS is More Accurate to Monitor Intensity than Heart Rate. International journal of sports physiology and performance, 12, 440-447. 
Butcher, S. J., Neyedly, T. J., Horvey, K. J., \& Benko, C. R. (2015). Do physiological measures predict selected CrossFit( $\left(^{\oplus}\right)$ benchmark performance? Open Access Journal of Sports Medicine, 6, 241-247.

Corrà, U., Agostoni, P. G., Anker, S. D., Coats, A. J. S., Crespo Leiro, M. G., de Boer, R. A., ... Piepoli, M. F. (2018). Role of cardiopulmonary exercise testing in clinical stratification in heart failure. A position paper from the Committee on Exercise Physiology and Training of the Heart Failure Association of the European Society of Cardiology. European Journal of Heart Failure, 20(1), 3-15.

Crum, E. M., O'Connor, W. J., Van Loo, L., Valckx, M., \& Stannard, S. R. (2017). Validity and reliability of the Moxy oxygen monitor during incremental cycling exercise. European Journal of Sport Science, 17(8), 1037-1043.

Dexheimer, J. D., Schroeder, E. T., Sawyer, B. J., Pettitt, R. W., Aguinaldo, A. L., \& Torrence, W. A. (2019). Physiological Performance Measures as Indicators of CrossFit ${ }^{\oplus}$ Performance. Sports (Basel, Switzerland), 7(4).

Drum, S. N., Bellovary, B. N., Jensen, R. L., Moore, M. T., \& Donath, L. (2017). Perceived demands and postexercise physical dysfunction in CrossFit ${ }^{\circledR}$ compared to an ACSM based training session. The Journal of Sports Medicine and Physical Fitness, 57(5), 604-609.

Feito, Y., Heinrich, K. M., Butcher, S. J., \& Poston, W. S. C. (2018). High-Intensity Functional Training (HIFT): Definition and Research Implications for Improved Fitness. Sports (Basel, Switzerland), 6(3).

Fernández, J. F., Solana, R. S., Moya, D., Marin, J. M. S., \& Ramón, M. M. (2015). Acute physiological responses during crossfit ${ }^{\oplus}$ workouts. European Journal of Human Movement, 35(0), 114-124.

Ferrari, M., \& Quaresima, V. (2012). A brief review on the history of human functional near-infrared spectroscopy (fNIRS) development and fields of application. Neurolmage, 63(2), 921-935.

Fryer, S., Stoner, L., Scarrott, C., Lucero, A., Witter, T., Love, R., ... Draper, N. (2015). Forearm oxygenation and blood flow kinetics during a sustained contraction in multiple ability groups of rock climbers. Journal of Sports Sciences, 33(5), 518-526.

Glasman, G. (2007). Understanding CrossFit. The CrossFit Journal, 56, 1-2.

Guyenet, P. G., \& Bayliss, D. A. (2015). Neural control of breathing and CO2 homeostasis. Neuron, 87(5), 946-961.

HajGhanbari, B., Yamabayashi, C., Buna, T. R., Coelho, J. D., Freedman, K. D., Morton, T. A., ... Reid, W. D. (2013). Effects of respiratory muscle training on performance in athletes: a systematic review with meta-analyses. Journal of Strength and Conditioning Research, (276), 1643-1663.

Heavens, K. R., Szivak, T. K., Hooper, D. R., Dunn-Lewis, C., Comstock, B. A., Flanagan, S. D., .. Kraemer, W. J. (2014). The effects of high intensity short rest resistance exercise on muscle damage markers in men and women. Journal of Strength and Conditioning Research, 28(4), 1041-1049.

Chance, B., Dait, M. T., Zhang, C., Hamaoka, T., \& Hagerman, F. (1992). Recovery from exercise-induced desaturation in the quadriceps muscles of elite competitive rowers. The American Journal of Physiology, 262(3 Pt 1), C766-775.

Jiménez Morgan, S., \& Molina Mora, J. A. (2017). Effect of Heart Rate Variability Biofeedback on Sport Performance, a Systematic Review. Applied Psychophysiology and Biofeedback, 42(3), 235-245.

Kliszczewicz, B., Quindry, C. J., Blessing, L. D., Oliver, D. G., Esco, R. M., \& Taylor, J. K. (2015). Acute Exercise and Oxidative Stress: CrossFit(TM) vs. Treadmill Bout. Journal of Human Kinetics, 47, 81-90.

Maté-Muñoz, J. L., Lougedo, J. H., Barba, M., Cañuelo-Márquez, A. M., Guodemar-Pérez, J., García-Fernández, P., ... GarnachoCastaño, M. V. (2018). Cardiometabolic and Muscular Fatigue Responses to Different CrossFit ${ }^{\oplus}$ Workouts. Journal of Sports Science \& Medicine, 17(4), 668-679.

McManus, C. J., Collison, J., \& Cooper, C. E. (2018). Performance comparison of the MOXY and PortaMon near-infrared spectroscopy muscle oximeters at rest and during exercise. Journal of Biomedical Optics, 23(1), 1-14.

Perciavalle, V., Marchetta, N. S., Giustiniani, S., Borbone, C., Perciavalle, V., Petralia, M. C., ... Coco, M. (2016). Attentive processes, blood lactate and CrossFit ${ }^{\oplus}$. The Physician and Sportsmedicine, 44(4), 403-406.

Perrey, S., \& Ferrari, M. (2018). Muscle Oximetry in Sports Science: A Systematic Review. Sports Medicine, 48(3), 597-616.

Tibana, R. A., de Almeida, L. M., Frade de Sousa, N. M., Nascimento, D. da C., Neto, I. V. de S., de Almeida, J. A., ... Prestes, J. (2016). Two Consecutive Days of Crossfit Training Affects Pro and Anti-inflammatory Cytokines and Osteoprotegerin without Impairments in Muscle Power. Frontiers in Physiology, 7, 260.

Wonisch, M., Hofmann, P., Pokan, R., Kraxner, W., Hödl, R., Maier, R., ... Fruhwald, F. M. (2003). Spiroergometry in cardiology - Physiology and terminology. Journal fur Kardiologie, 10, 383-390. 